Archive for

Organic Chemistry

Arkivoc 2020, part iv, 35-49

\title{
Synthesis of arylbenziodoxoles using pseudocyclic benziodoxole triflate and arenes
}

\author{
Akira Yoshimura, ${ }^{* a, b}$ Scott M. Larson, ${ }^{a}$ Gunnar B. Frahm, ${ }^{a}$ Christopher D. Huss, ${ }^{\text {a }}$ Gregory T. Rohde, ${ }^{c}$ Victor N. \\ Nemykin, ${ }^{d}$ Mekhman S. Yusubov, ${ }^{b}$ Akio Saito, ${ }^{e}$ and Viktor V. Zhdankin \\ ${ }^{a}$ Department of Chemistry and Biochemistry, University of Minnesota Duluth, Duluth, Minnesota 55812, USA \\ ${ }^{b}$ Tomsk Polytechnic University, 634050 Tomsk, Russia \\ ${ }^{c}$ Marshall School, Duluth, Minnesota 55811, USA \\ ${ }^{d}$ Department of Chemistry, University of Manitoba, Winnipeg, MB R3T 2N2, Canada \\ ${ }^{e}$ Division of Applied Chemistry, Institute of Engineering, Tokyo University of Agriculture and Technology, \\ 2-23-16 Naka-cho, Koganei, Tokyo 184-8588, Japan \\ Email: ayoshimu@d.umn.edu
}

Received 08-05-2020

Accepted 09-12-2020

Published on line $09-24-2020$

\section{Abstract}

An acid activated pseudocyclic hypervalent iodine reagent, 2-[hydroxy(trifluoromethanesufonyloxy)]iodobenzoic acid, can easily react with various arenes in the presence of trifluoromethanesulfonic acid to produce pseudocyclic diaryliodonium triflate salts. This synthetic procedure proceeds under mild conditions to afford the respective iodonium salts in moderate to good yields. Several pseudocyclic diaryliodonium triflate salts structures have been confirmed by X-ray crystallography. Obtained products can be easily converted to cyclic hypervalent iodine(III) compounds, arylbenziodoxolones, in moderate to good yields under basic conditions.<smiles>O=C1O[I-](O)([OH+])c2ccccc21</smiles>

IBA-TfOH<smiles></smiles>

Pseudocyclic Arl(III)

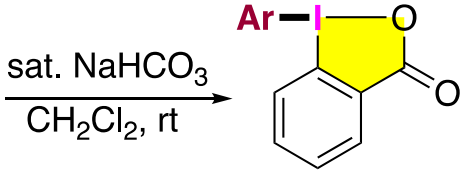

Cyclic ArI(III)

Keywords: Hypervalent iodine reagent, pseudocyclic diaryliodonium salts, arylbenziodoxolones, benziodoxole, cyclization 


\section{Introduction}

Hypervalent iodine compounds have been used as efficient oxidizing reagents and employed in many conversion reactions in organic synthesis. Diaryliodonium salts are one of the most important classes of hypervalent iodine(III) reagents, and many attractive reactions using them have been reported. ${ }^{1-11}$ In particular, most of these compounds are commonly used as the electrophilic aryl transfer reagents toward various organic substrates, resulting in the synthesis of various aryl derivatives. ${ }^{12-17}$ Diaryliodonium salts are also used as benzyne precursor reagents, and many benzyne-mediated reactions utilizing these reagents have been reported. ${ }^{18,19}$ While numerous reaction examples using diaryliodonium salts are well-known, a number of synthetic studies on them have also been reported. ${ }^{12} \mathrm{~A}$ typical synthetic method of diaryiodonium salts is to combine common hypervalent iodine(III) reagents with aromatic compounds under appropriate conditions. As a developed synthetic example, the diaryliodonium compounds can be prepared from iodoarenes and aromatic compounds by treatment with suitable oxidants.

Recently, various pseudocyclic hypervalent iodine(III) compounds have been prepared and investigated. ${ }^{20,21}$ Most of these compounds have higher stability and improved reactivity in comparison to their respective non-cyclic hypervalent iodine(III) reagents. ${ }^{22-36}$ Previously, our group has reported the preparation, structure, and reactivity of 2-[hydroxy(trifluoromethanesulfonyloxy)]-iodobenzoic acid (IBA$\mathrm{TfOH}$ ), which is the pseudocylcic analogue compound of [hydroxy(trifluoromethanesulfonyloxy)iodo]benzene Phl(OH)OTf. ${ }^{37-40}$ The novel IBA-TfOH reagent showed better reactivity as well as higher stability than $\mathrm{Phl}(\mathrm{OH}) \mathrm{OTf}$. In our previous experiment, the reactivity of IBA-TfOH using mesitylene was investigated as an efficient approach to the pseudocyclic diaryliodonium triflate (Scheme 1$).{ }^{37}$

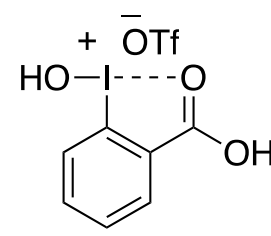

(1 equiv)

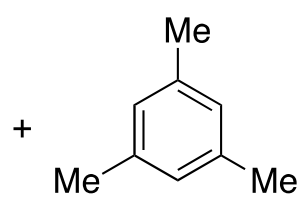

(1 equiv)

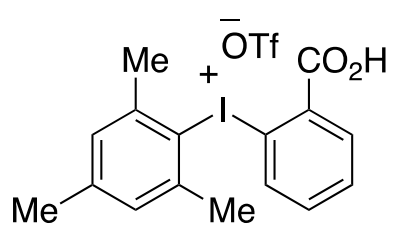

$89 \%$

Scheme 1. Reaction of IBA-TfOH using mesitylene.

However, the reactivity towards other arenes using IBA-TfOH has not been reported. In the present study, we report the optimization and scope for the preparation of pseudocyclic diaryliodonium triflates from IBA$\mathrm{TfOH}$ reagents with various arenes in the presence of trifluoromethanesulfonic acid. The obtained products can be further converted to the cyclic hypervalent iodine(III) compounds, arylbenziodoxolones, under mild basic conditions. ${ }^{41,42}$

\section{Results and Discussion}

We investigated the preparation of pseudocyclic diaryliodonium triflate $\mathbf{3 a}$ using IBA-TfOH $\mathbf{1}$ and benzene $\mathbf{2} \mathbf{a}$ in different solvents based on our previous results (Table 1). In the initial study, the reaction was performed in 2,2,2-trifluoroethanol (TFE) in the absence of TfOH under our previously reported condition resulting in no desired product 3a, and the reagent $\mathbf{1}$ was recovered from the reaction mixture (entry 1 ). The reaction using 
excess amount of benzene $\mathbf{2 a}$ in TFE or dichloromethane also did not produce the desired product $\mathbf{3 a}$ (entries 2 and 3). Addition of TfOH as an additive in dichloromethane dramatically improved the reaction to give the desired product $3 a$ in quantitative yield (entry 4). Screening of various solvents in the presence of TfOH has demonstrated that dichloromethane is the best solvent in this transformation reaction (entries 4-10). Decreasing the amount of benzene $2 \mathrm{a}$ from 56.0 to 4.0 equivalents did not affect the yield of product $3 a$ (entries 4, 11-13). Further decreasing the amount of benzene $\mathbf{2 a}$ led to a reduced yield of product $3 a$ (entries 14-16).

Table 1. Optimization of synthesis of pseudocyclic diaryliodonium triflate $3 \mathbf{a}^{\mathrm{a}}$
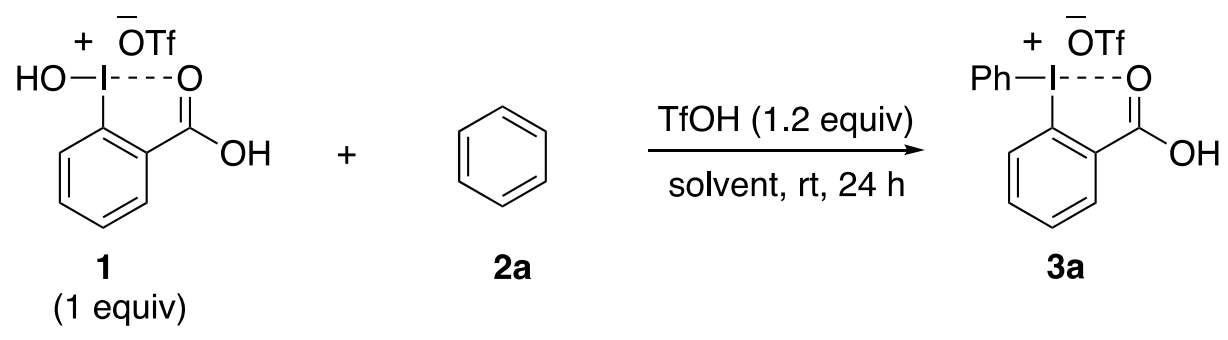

\begin{tabular}{llll}
\hline Entry & Benzene 2a (equiv.) & Solvent & 3a (\%) \\
\hline $1^{\mathrm{c}}$ & 1 & $\mathrm{TFE}$ & $0^{\mathrm{d}}$ \\
$2^{\mathrm{c}}$ & 56 & $\mathrm{TFE}$ & $0^{\mathrm{d}}$ \\
$3^{\mathrm{c}}$ & 56 & $\mathrm{CH}_{2} \mathrm{Cl}_{2}$ & $0^{\mathrm{d}}$ \\
4 & 56 & $\mathrm{CH}_{2} \mathrm{Cl}_{2}$ & $>99$ \\
5 & 56 & $\mathrm{TFE}$ & $0^{\mathrm{d}}$ \\
6 & 56 & $\mathrm{MeCN}$ & $0^{\text {d }}$ \\
7 & 56 & $\mathrm{AcOEt}$ & $0^{\mathrm{d}}$ \\
8 & 56 & $\mathrm{Heptane}$ & $0^{\mathrm{d}}$ \\
9 & 56 & $\mathrm{ClCH}_{2} \mathrm{CH}_{2} \mathrm{Cl}$ & 80 \\
10 & 56 & $\mathrm{CHCl}_{3}$ & 82 \\
11 & 10 & $\mathrm{CH}_{2} \mathrm{Cl}_{2}$ & $>99$ \\
12 & 5 & $\mathrm{CH}_{2} \mathrm{Cl}_{2}$ & $>99$ \\
13 & 4 & $\mathrm{CH}_{2} \mathrm{Cl}_{2}$ & $>99$ \\
14 & 3 & $\mathrm{CH}_{2} \mathrm{Cl}_{2}$ & 84 \\
15 & 2 & $\mathrm{CH}_{2} \mathrm{Cl}_{2}$ & 79 \\
16 & 1 & $\mathrm{CH}_{2} \mathrm{Cl}_{2}$ & 58 \\
\hline
\end{tabular}

${ }^{a}$ Reaction conditions: IBA-TfOH 1 (1 equiv.), benzene 2a (1-56 equiv.) and TfOH (0-1.2 equiv.) in various solvent $(2.0 \mathrm{~mL})$ at room temperature for 24 hours. ${ }^{b}$ Yield of isolated product. ${ }^{c}$ In the absence of TfOH. ${ }^{d}$ IBA$\mathrm{TfOH} 1$ was recovered from the reaction mixture.

By using the optimal conditions, we have investigated the conversion of various substituted arenes 2 to the respective pseudocyclic diaryliodonium triflates $\mathbf{3}$. In general, the reaction of arenes with either electrondonating or electron-withdrawing substituents gave the corresponding desired products $\mathbf{3 a - 1}$ in moderate to good yields. The reaction with sterically bulky ortho-substituted arenes also gave the pseudocyclic diaryliodonium triflates $\mathbf{3}$ in moderate to good yields (Table 2). Structures of pseudocyclic diaryliodonium triflates 3a, 3f and $\mathbf{3 k}$ were established by $\mathbf{X}$-ray crystallography (Figure 2). According to the X-ray data, the 
pseudocyclic diaryliodonium structures with strong intramolecular interaction between iodine and oxygen atoms were observed. ${ }^{20,30,31,37,43}$ The triflate oxygen atom was also involved in a weak intermolecular interaction with iodine atom resulting in a pseudo-square planar coordination of hypervalent iodine center.

Table 2. Preparation of pseudocyclic diaryliodonium triflates $\mathbf{3}$ using IBA-TfOH $\mathbf{1}$ with various arene $\mathbf{2}^{\mathrm{a}}$<smiles>CO[I-]([O-])(O)c1ccccc1C(=O)O</smiles>

1

(1 equiv)

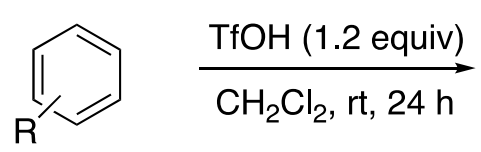

2

(4 equiv)<smiles>O=C(O)c1ccccc1[I+]([O-])c1ccccc1</smiles>

3a-m<smiles>O=C(O)c1ccccc1[I-]c1ccccc1</smiles>

3a $99 \%$<smiles>CC[C+]([I+]c1ccc(C(C)(C)C)cc1)c1ccccc1C(=O)O</smiles>

3e $100 \%$<smiles>CC[18O]c1ccccc1[I+]([O+])c1ccc(OC)cc1</smiles>

3i $100 \%$<smiles>CC[O+]c1c(C)cc(C)cc1C(=O)O</smiles>

3b $100 \%$<smiles>CC[O+][I-](c1ccc(C)cc1)c1ccccc1C(=O)O</smiles>

3f $97 \%$<smiles>CCOc1ccc([I+]([OH2+])c2ccccc2C(=O)O)cc1</smiles>

3j $97 \%$<smiles>CC[O+][I-]c1cc(C)ccc1C</smiles>

3c $94 \%$<smiles>Cc1cc(C(C)C)cc(C(C)C)c1[In](c1ccccc1C(=O)O)c1c(C(C)C)cc(C(C)C)cc1C(C)C</smiles>

3g $100 \%$<smiles>COc1cc(OC)c(I(c2ccccc2C(=O)O)[I+](=O)[O-])c(OC)c1</smiles>

3k $64 \%$<smiles>Cc1cc(C)c(C)c([I+]([O-])c2ccccc2C(=O)O)c1C</smiles><smiles>CCc1ccc(I([OH2+])c2ccccc2C(=O)O)cc1CC</smiles>

3h $93 \%$

a Reaction conditions: IBA-TfOH 1 (1 equiv.), arene 2 (4 equiv.) and TfOH (1.2 equiv.) in dichloromethane at room temperature for 24 hours. 

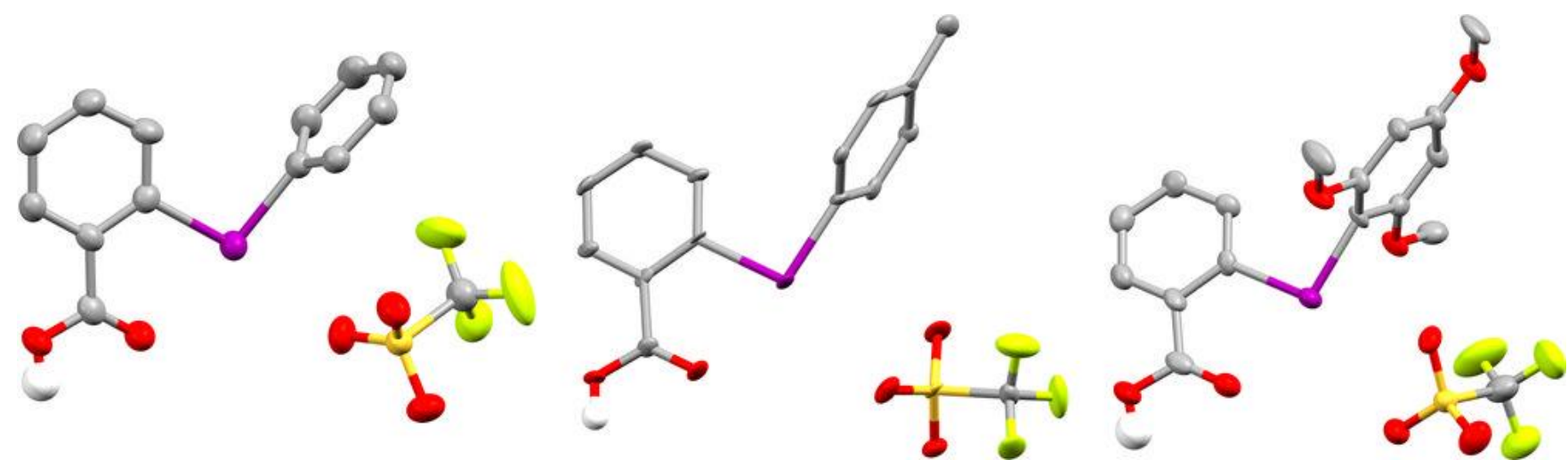

Figure 1. X-ray crystal structure of 3a (CCDC 2021394), 3f (CCDC 2021395) and 3k (CCDC 2021393). Thermal Ellipsoids were drawn at the $50 \%$ probability level. Hydrogen atoms bonded to carbon atoms and water molecules in 3a and 3f were removed for clarity. lodine-oxygen close contact distances in $\AA$ : 3a $1 \cdots O(B A)=$

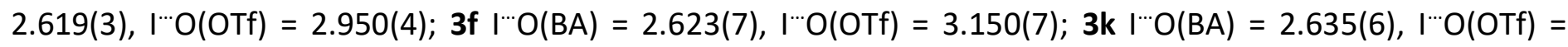
2.838(6).

Next, we investigated a one-pot preparation of pseudocyclic diaryliodonium triflate $\mathbf{3 a}$ from benzene $\mathbf{2 a}$ and IBA-TfOH 1 generated in situ from 2-iodosylbenzoic acid 4 a with trifluoromethanesulfonic acid (Scheme 2). This reaction gave the pseudocyclic diaryliodonium triflate $3 a$ in quantitative yield (eq. 1). ${ }^{37}$ Compared to the previously reported method for preparation of pseudocyclic diaryliodonium triflates, our one-pot procedure was able to afford these products in comparable yields. ${ }^{44}$ As expected, the reaction of substituted iodosylbenzoic acids $\mathbf{4 b}, \mathbf{c}$ under one-pot method resulted in the corresponding compounds $\mathbf{3 m}, \mathbf{n}$ in good yields (eq. 1). ${ }^{45}$ Furthermore, we have found that 3 a could be prepared in moderate yield from 2-iodobenzoic acid and benzene using $m$-chloroperoxybenzoic acid in the presence of trifluoromethanesulfonic acid (eq. 2). ${ }^{41}$<smiles>[Y4]c1ccc2c(c1)C(=O)OI2O</smiles>

(1 equiv) $(\mathrm{X}=\mathrm{H}, \mathrm{Me}, \mathrm{Br})$<smiles>O=C(O)c1ccccc1I</smiles>

(1 equiv)

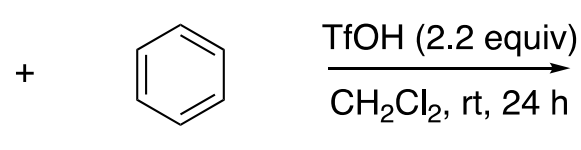

2a

(4 equiv)<smiles>c1ccccc1</smiles>

2a

(4 equiv)<smiles>[X]c1ccc([I-][O+]=[OH+])c(C(=O)O)c1</smiles>

(eq. 1)

$$
\begin{aligned}
& X=\mathrm{H} ; 3 \mathbf{3} 100 \% \\
& X=\mathrm{Me} ; 3 \mathbf{3 m ~ 9 2 \%} \\
& X=\mathrm{Br} ; 3 \mathbf{3 n} 84 \%
\end{aligned}
$$<smiles>O=C(O)c1ccccc1[I-][Te](c1ccccc1)c1ccccc1</smiles>

3a $68 \%$

Scheme 2. One-pot synthesis of pseudocyclic diaryliodonium triflates.

Finally, we have demonstrated that the obtained pseudocyclic diaryliodonium triflates could be converted to arylbenziodoxolones. ${ }^{41,42,46,47}$ In particular, the treatment of 3 a with sodium bicarbonate could lead to phenylbenziodoxolone $\mathbf{6 a}$ in quantitative yield. Following this strategy, we performed the reaction of 
substituted pseudocyclic diaryliodonium triflates 3a-n resulting in the desired arylbenziodoxolones 6a-n in moderate to good yields (Table 3).

Table 3. Preparation of arylbenziodoxolones 6 from pseudocyclic diaryliodonium triflates $\mathbf{3}^{\mathrm{a}}$
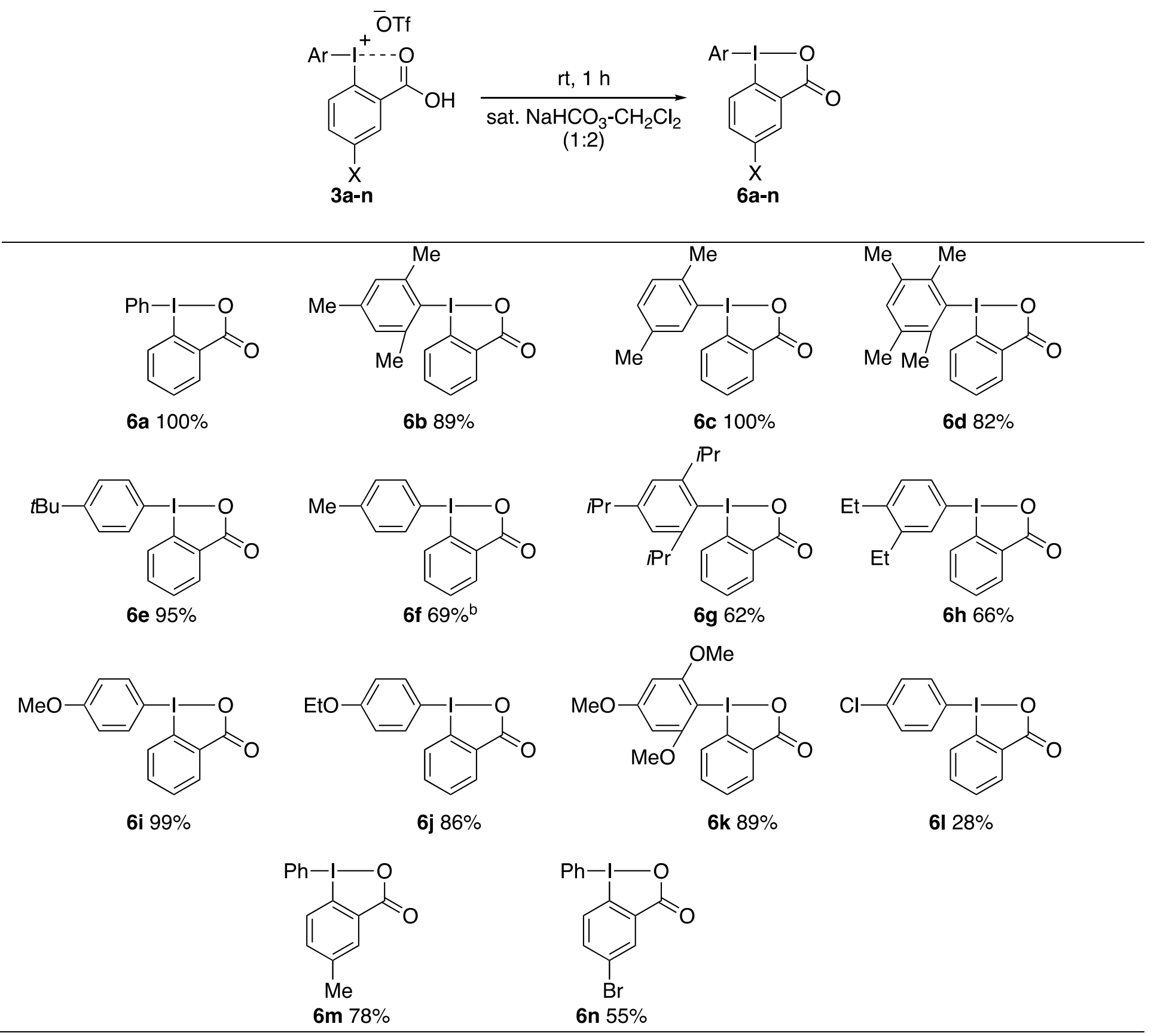

${ }^{a}$ Reaction conditions: Pseudocyclic diaryliodonium triflates 3 in saturated $\mathrm{NaHCO}_{3}$ aqueous -dichloromethane $(1: 2)$ at room temperature for 1 hour. ${ }^{b}$ The isomeric ortho and para products were detected.

\section{Conclusions}

In summary, we have prepared the pseudocyclic diaryliodonium triflates from IBA-TfOH and arenes in the presence of $\mathrm{TfOH}$. The structure of several products $\mathbf{3}$ was confirmed by X-ray crystallography. The 
combination of 2-iodosylbenzoic acid and trifluoromethanesulfonic acid, or 2-iodobenzoic acid and $\mathrm{m}$ chloroperoxybenzoic acid in the presence of trifluoromethanesulfonic acid generates IBA-TfOH in-situ, which can be used for the preparation of pseudocyclic diaryliodonium triflates. Furthermore, the produced pseudocyclic diaryliodonium triflates can be easily converted to the respective arylbenziodoxolones under mild basic condition.

\section{Experimental Section}

General. All reactions were performed under dry argon atmosphere with flame-dried glassware. Dichloromethane was distilled from $\mathrm{CaH}_{2}$ immediately prior to use. All commercial reagents were ACS reagent grade and used without further purification. Melting points were determined in an open capillary tube with a Mel-temp II melting point apparatus. Infrared spectra were recorded on a PerkinElmer 1600 series FT-IR spectrophotometer. NMR spectra were recorded on a Varian Inova 500, $300 \mathrm{MHz}$ and Bruker $400 \mathrm{MHz} \mathrm{NMR}$ spectrometer $\left({ }^{1} \mathrm{H}\right.$ NMR and ${ }^{13} \mathrm{C}$ NMR). X-ray crystal analysis was performed by Rigaku RAPID II XRD Image Plate using graphite-monochromated $\mathrm{Cu}$ or Mo K $\alpha$ radiation ( $(\lambda=1.54187$ or $0.71073 \AA)$ at 125 or $173 \mathrm{~K}$. Please see the cif file for more detailed crystallography information. Hypervalent iodine reagents, IBA-TfOH $1,{ }^{39}, 2-$ lodosylbenzoic acids $\mathbf{4 a -} \mathbf{b}^{48}$, and $\mathbf{4} \mathbf{c}^{49}$ were prepared according to the reported procedure.

\section{General procedure for preparation of pseudocyclic diaryliodonium triflates $\mathbf{3}$ using IBA-TfOH 1 and arenes 2.} Arenes $2(0.80 \mathrm{mmol})$, and trifluoromethanesulfonic acid $(30 \mathrm{mg}, 0.20 \mathrm{mmol}$ ) were added to a solution of IBA$\mathrm{TfOH} 1(83 \mathrm{mg}, 0.20 \mathrm{mmol})$ in dichloromethane $(1 \mathrm{~mL})$. The reaction was stirred at room temperature for 24 hours. After completion of the reaction, the solvent was removed under reduced pressure and the solid product was washed with diethyl ether several times then dried in vacuum to give the pure compound 3.

2-Carboxyphenyl(phenyl)iodonium triflate (3a). ${ }^{44}$ Reaction of benzene $2 \mathrm{2a}(62 \mathrm{mg}, 0.80 \mathrm{mmol}$ ) according to general procedure afforded $98 \mathrm{mg}(99 \%)$ of product $3 a$ isolated as a white solid: $\mathrm{mp} 230.5-231.4{ }^{\circ} \mathrm{C}$ (lit. ${ }^{44}$; mp $\left.198-220^{\circ} \mathrm{C}\right)$; IR (KBr) cm ${ }^{-1} 3479,3075,3052,1674,1586,1472,1444,1292,1163,1023,911,745 ;{ }^{1} \mathrm{H}$ NMR (300 $\left.\mathrm{MHz}, \mathrm{CD}_{3} \mathrm{CN}\right): \delta 8.40(\mathrm{dd}, J 7.5 \mathrm{~Hz}, 1.5 \mathrm{~Hz}, 1 \mathrm{H}), 8.17(\mathrm{~d}, J 8.4 \mathrm{~Hz}, 2 \mathrm{H}), 7.96(\mathrm{t}, J 7.5 \mathrm{~Hz}, 1 \mathrm{H}), 7.84-7.66(\mathrm{~m}, 4 \mathrm{H})$, $7.07(\mathrm{~d}, J 8.4 \mathrm{~Hz}, 1 \mathrm{H}) ;{ }^{13} \mathrm{C} \mathrm{NMR}\left(75 \mathrm{MHz}, \mathrm{CD}_{3} \mathrm{CN}\right): \delta$ 169.0, 138.1, 137.5, 134.1, 133.3, 132.7, 131.7, 129.7, 126.3, $120.9\left(q,{ }^{1} J_{C F}=318.2 \mathrm{~Hz}\right), 114.3,109.2 ;{ }^{19} \mathrm{~F} \mathrm{NMR}\left(282 \mathrm{MHz}, \mathrm{CD}_{3} \mathrm{CN}\right): \delta-79.4 ; \mathrm{HRMS}$ (APCl-positive ionization): calcd for $\mathrm{C}_{13} \mathrm{H}_{10} \mathrm{IO}_{2}$ ([M-OTf $\left.]^{+}\right)$: 324.9725 , found: 324.9741 .

Single crystals of product 3a suitable for X-ray crystallographic analysis were obtained by slow evaporation of acetonitrile solution. For details on crystal structure of compound $3 a$ see the CIF file in Supporting Information. Selected crystallographic data for 3a: Monoclinic, P21n, a = 11.5802(10) $\AA, b=9.0047(10)) \AA, c=$ 16.6435(18), $\beta=95.433(7) \AA ̊ ., V=1727.7(3) \AA ̊ 3, Z=4, R(I>2.0 / \sigma(I))=0.0469, \mathrm{Rw}(\mathrm{all})=0.0760, \mathrm{CCDC} 2021394$.

2-Carboxyphenyl(mesityl)iodonium triflate (3b). ${ }^{37}$ Reaction of mesitylene $\mathbf{2 b}$ (96 $\mathrm{mg}, 0.80 \mathrm{mmol}$ ) according to general procedure afforded $103 \mathrm{mg}(100 \%)$ of product $\mathbf{3 b}$ isolated as a white solid: $\mathrm{mp} 187.6-190.5^{\circ} \mathrm{C}$ (lit. ${ }^{37}$; $\left.\mathrm{mp} 185.3-186.6^{\circ} \mathrm{C}\right)$; IR (KBr) cm ${ }^{-1} 3456,3073,3026,1684,1585,1465,1440,1289,1169,1034,990,750 ;{ }^{1} \mathrm{H}$ NMR $\left(300 \mathrm{MHz}, \mathrm{CD}_{3} \mathrm{CN}\right): \delta 8.38(\mathrm{~d}, J 7.5 \mathrm{~Hz}, 1 \mathrm{H}), 7.77(\mathrm{t}, J 7.5 \mathrm{~Hz}, 1 \mathrm{H}), 7.70-7.62(\mathrm{~m}, 1 \mathrm{H}), 7.84-7.66(\mathrm{~m}, 4 \mathrm{H})$, $7.34(\mathrm{~s}, 2 \mathrm{H}), 6.93(\mathrm{t}, J 8.1 \mathrm{~Hz}, 1 \mathrm{H}), 2.50(\mathrm{~s}, 6 \mathrm{H}), 2.44(\mathrm{~s}, 3 \mathrm{H})$; HRMS (ESI-positive ionization): calcd for $\mathrm{C}_{16} \mathrm{H}_{16} \mathrm{IO}_{2}$ $\left([\mathrm{M}-\mathrm{OTf}]^{+}\right): 367.0195$, found: 367.0205 .

2-Carboxyphenyl(2,5-dimethylphenyl)iodonium triflate (3c). Reaction of 1,4-dimethylbenzene 2c (75 mg, $0.80 \mathrm{mmol}$ ) according to general procedure afforded $94 \mathrm{mg}(94 \%)$ of product $3 \mathrm{c}$ isolated as a white solid: $\mathrm{mp}$ $175.5-176.7^{\circ} \mathrm{C}$; IR (KBr) cm ${ }^{-1} 3504,3078,3052,2928,1673,1586,1492,1470,1442,1280,1162,1029,754 ;{ }^{1} \mathrm{H}$ 
NMR (300 MHz, CD ${ }_{3} \mathrm{CN}$ ): $\delta 8.38$ (dd, J $\left.7.5 \mathrm{~Hz}, 1.8 \mathrm{~Hz}, 1 \mathrm{H}\right), 7.96(\mathrm{~s}, 1 \mathrm{H}), 7.77(\mathrm{t}, J 7.5 \mathrm{~Hz}, 1 \mathrm{H}), 7.72-7.59(\mathrm{~m}, 3 \mathrm{H})$, $6.98(\mathrm{~d}, J 8.4 \mathrm{~Hz}, 1 \mathrm{H}), 2.48(\mathrm{~s}, 3 \mathrm{H}), 2.40(\mathrm{~s}, 3 \mathrm{H}) ;{ }^{13} \mathrm{C} \mathrm{NMR}\left(100 \mathrm{MHz}, \mathrm{CD}_{3} \mathrm{CN}\right): \delta 169.5,141.2,141.1,140.1,138.1$, 136.0, 134.0, 132.4, 132.3, 129.6, 127.3, 121.5 (q, $\left.{ }^{1} J_{C F}=318.3 \mathrm{~Hz}\right), 114.3,113.5,24.5,20.2 ;{ }^{19} \mathrm{~F} \mathrm{NMR}(377 \mathrm{MHz}$, $\mathrm{CD}_{3} \mathrm{CN}$ ): $\delta$-79.3; HRMS (APCI-positive ionization): calcd for $\mathrm{C}_{15} \mathrm{H}_{14} \mathrm{IO}_{2}$ ([M-OTf] ${ }^{+}$): 353.0038 , found: 353.0056 .

2-Carboxyphenyl(2,3,5,6-tetramethylphenyl)iodonium triflate (3d). Reaction of 1,2,4,5-tetramethylbenzene 2d (107 $\mathrm{mg}, 0.80 \mathrm{mmol}$ ) according to general procedure afforded $100 \mathrm{mg}$ (94\%) of product $\mathbf{3 d}$ isolated as a white solid: $\mathrm{mp} 165.4-166.2{ }^{\circ} \mathrm{C}$; IR $(\mathrm{KBr}) \mathrm{cm}^{-1}$ 3431, 3050, 2985, 2927, 1676, 1588, 1472, 1443, 1287, 1161, 1024, 743; ${ }^{1} \mathrm{H}$ NMR (300 MHz, CD $\left.{ }_{3} \mathrm{CN}\right): \delta 8.38$ (dd, J 7.5 Hz, $\left.1.8 \mathrm{~Hz}, 1 \mathrm{H}\right), 7.80-7.77(\mathrm{~m}, 1 \mathrm{H}), 7.64$ (td, J 7.5 Hz, 1.5 $\mathrm{Hz}, 1 \mathrm{H}), 7.44(\mathrm{~s}, 1 \mathrm{H}), 6.93(\mathrm{~d}, J 8.1 \mathrm{~Hz}, 1 \mathrm{H}), 2.48(\mathrm{~s}, 6 \mathrm{H}), 2.40(\mathrm{~s}, 6 \mathrm{H}) ;{ }^{13} \mathrm{C} \mathrm{NMR}\left(75 \mathrm{MHz}, \mathrm{CD}_{3} \mathrm{CN}\right): \delta 168.7,139.9$, 137.5, 137.4, 133.6, 131.7, 128.7, 122.1, 120.1 (q, $\left.{ }^{1} J_{C F}=321.1 \mathrm{~Hz}\right), 112.1,110.0,23.6,20.6 ;{ }^{19} \mathrm{~F} \mathrm{NMR}(282 \mathrm{MHz}$, $\mathrm{CD}_{3} \mathrm{CN}$ ): $\delta$-79.3; HRMS (APCI-positive ionization): calcd for $\mathrm{C}_{17} \mathrm{H}_{18} \mathrm{IO}_{2}$ ([M-OTf] ${ }^{+}$): 381.0351, found: 381.0355 .

2-Carboxyphenyl(4-tert-butylphenyl)iodonium triflate (3e). Reaction of tert-butylbenzene 2e (107 mg, 0.80 mmol) according to general procedure afforded $106 \mathrm{mg}(100 \%)$ of product $3 \mathrm{e}$ isolated as a white solid: $\mathrm{mp}$ 172.1-175.0 ${ }^{\circ} \mathrm{C}$; IR (KBr) cm ${ }^{-1} 3466,3085,2964,2910,1655,1585,1469,1438,1260,1171,1030,755 ;{ }^{1} \mathrm{H} \mathrm{NMR}$ $\left(500 \mathrm{MHz}, \mathrm{CD}_{3} \mathrm{CN}\right): \delta 8.37(\mathrm{~d}, J 8.0 \mathrm{~Hz}, 1 \mathrm{H}), 8.02(\mathrm{~d}, J 8.0 \mathrm{~Hz}, 1 \mathrm{H}), 7.78-7.71(\mathrm{~m}, 3 \mathrm{H}), 7.02(\mathrm{~d}, J 8.5 \mathrm{~Hz}, 1 \mathrm{H}), 1.40$ $(\mathrm{s}, 9 \mathrm{H}) ;{ }^{13} \mathrm{C}$ NMR $\left(100 \mathrm{MHz}\right.$, DMSO- $\left.d_{6}\right): \delta 169.1,156.9,137.8,136.5,132.9,131.5,130.0,129.8,121.2,\left(\mathrm{q},{ }^{1} J_{C F}=\right.$ $320.0 \mathrm{~Hz}$ ), 116.34, 109.9, 35.6, 31.3; ${ }^{19} \mathrm{~F} \mathrm{NMR} \mathrm{(377} \mathrm{MHz,} \mathrm{DMSO-} d_{6}$ ): $\delta-77.7$; HRMS (APCI-positive ionization): calcd for $\mathrm{C}_{17} \mathrm{H}_{18} \mathrm{IO} 2\left([\mathrm{M}-\mathrm{OTf}]^{+}\right): 381.0351$, found: 381.0347 .

2-Carboxyphenyl(4-methylphenyl)iodonium triflate (3f). ${ }^{44}$ Reaction of toluene $2 \mathrm{f}$ (74 $\left.\mathrm{mg}, 0.80 \mathrm{mmol}\right)$ according to general procedure afforded $95 \mathrm{mg}$ (97\%) of product $3 \mathrm{f}$ isolated as a white solid: $\mathrm{mp}$ 202.1-203.6 ${ }^{\circ} \mathrm{C}$; (lit. ${ }^{44}$; mp 193-194 $\left.{ }^{\circ} \mathrm{C}\right)$; IR (KBr) cm ${ }^{-1} 3469,3089,2990,1670,1587,1472,1440,1255,1166,1029,754 ;{ }^{1} \mathrm{H}$ $\operatorname{NMR}\left(300 \mathrm{MHz}, \mathrm{CD}_{3} \mathrm{CN}\right): \delta 8.37$ (d, J $\left.6.9 \mathrm{~Hz}, 1 \mathrm{H}\right), 7.98(\mathrm{~d}, J 8.1 \mathrm{~Hz}, 2 \mathrm{H}), 7.82-7.64(\mathrm{~m}, 2 \mathrm{H}), 7.53(\mathrm{~d}, J 8.1 \mathrm{~Hz}, 2 \mathrm{H})$, $7.05(\mathrm{~d}, J 7.5 \mathrm{~Hz}, 1 \mathrm{H}), 2.53(\mathrm{~s}, 3 \mathrm{H})$; HRMS (APCl-positive ionization): calcd for $\mathrm{C}_{14} \mathrm{H}_{12} \mathrm{IO}_{2}\left([\mathrm{M}-\mathrm{OTf}]^{+}\right)$: 338.9882 , found: 338.9885 .

Single crystals of product $\mathbf{3 f}$ suitable for X-ray crystallographic analysis were obtained by slow evaporation of acetonitrile solution. For details on crystal structure of compound $\mathbf{3 f}$ see the CIF file in Supporting Information. Selected crystallographic data for 3f: Monoclinic, P21n, $a=11.2493(2) \AA, b=10.31970(10) \AA, c=15.9332(11)$ $\AA, \beta=104.159(7) V=1793.48(12) \AA ̊ 3, Z=4, R(I>2.0 / \sigma(I))=0.0658, R w$ (all) = 0.0844, CCDC 2021395.

2-Carboxyphenyl(2,4,6-triisopropylphenyl)iodonium triflate $\mathbf{( 3 g})$. Reaction of 1,3,5-triisopropylbenzene $\mathbf{2 g}$ (163 $\mathrm{mg}, 0.80 \mathrm{mmol}$ ) according to general procedure afforded $120 \mathrm{mg}$ (100\%) of product $\mathbf{3 g}$ isolated as a light

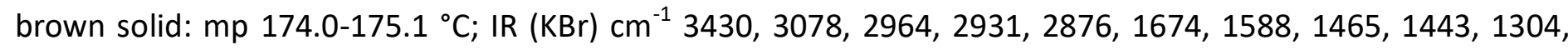
1170, 1024, 750; ${ }^{1} \mathrm{H}$ NMR (300 MHz, $\mathrm{CD}_{3} \mathrm{CN}$ ): $\delta 8.38$ (dd, J 7.5 Hz, $\left.1.8 \mathrm{~Hz}, 1 \mathrm{H}\right), 8.20$ (brs, $\left.1 \mathrm{H}\right), 7.80-7.63(\mathrm{~m}, 2 \mathrm{H})$, $7.45(\mathrm{~s}, 2 \mathrm{H}), 6.94(\mathrm{~d}, J 7.8 \mathrm{~Hz}, 1 \mathrm{H}), 3.24-3.20(\mathrm{~m}, 1 \mathrm{H}), 3.11-3.04(\mathrm{~m}, 2 \mathrm{H}), 1.33(\mathrm{~d}, J 6.9 \mathrm{~Hz}, 6 \mathrm{H}), 1.28-1.16(\mathrm{~m}$, $12 \mathrm{H}) ;{ }^{13} \mathrm{C} N M R\left(100 \mathrm{MHz}, \mathrm{CD}_{3} \mathrm{CN}\right): \delta 169.4,157.2,154.3,137.9,134.0,132.3,129.4,127.8,125.8,121.5\left(\mathrm{q},{ }^{1} J_{C F}\right.$ $=318.0 \mathrm{~Hz}$ ), 117.0, 114.4, 39.4, 34.8, 23.5; $\left.{ }^{19} \mathrm{~F} \mathrm{NMR} \mathrm{(282} \mathrm{MHz,} \mathrm{CD}{ }_{3} \mathrm{CN}\right): \delta$-79.3; HRMS (APCl-positive ionization): calcd for $\mathrm{C}_{22} \mathrm{H}_{28} \mathrm{IO}_{2}\left([\mathrm{M}-\mathrm{OTf}]^{+}\right)$: 451.1134 , found: 451.1136 .

2-Carboxyphenyl(3,4-diethylphenyl)iodonium triflate (3h). Reaction of 1,2-diethylbenzene $\mathbf{2 h}$ (107 $\mathrm{mg}, 0.80$ $\mathrm{mmol}$ ) according to general procedure afforded $99 \mathrm{mg}$ (93\%) of product $3 \mathrm{~h}$ isolated as a white solid: $\mathrm{mp} \mathrm{193.8-}$ $195.6{ }^{\circ} \mathrm{C}$; IR (KBr) cm ${ }^{-1} 3462,2974,2943,2880,1674,1586,1473,1439,1292,1176,1025,746 ;{ }^{1} \mathrm{H}$ NMR (500 $\left.\mathrm{MHz}, \mathrm{CD}_{3} \mathrm{CN}\right): \delta 8.37(\mathrm{dd}, J 8.0 \mathrm{~Hz}, 1.8 \mathrm{~Hz}, 1 \mathrm{H}), 7.91(\mathrm{~d}, J 2.3 \mathrm{~Hz}, 1 \mathrm{H}), 7.87(\mathrm{dd}, J 8.0 \mathrm{~Hz}, 2.3 \mathrm{~Hz}, 1 \mathrm{H}), 7.76(\mathrm{t}, J 8.0$ $\mathrm{Hz}, 1 \mathrm{H}), 7.69(\mathrm{t}, J 8.0 \mathrm{~Hz}, 1 \mathrm{H}), 7.51(\mathrm{~d}, J 8.0 \mathrm{~Hz}, 1 \mathrm{H}), 7.06(\mathrm{~d}, J 8.0 \mathrm{~Hz}, 1 \mathrm{H}), 2.83(\mathrm{q}, J 7.5 \mathrm{~Hz}, 2 \mathrm{H}), 2.78(\mathrm{q}, J 7.5 \mathrm{~Hz}$, $2 \mathrm{H}), 1.28(\mathrm{t}, J 7.5 \mathrm{~Hz}, 3 \mathrm{H}), 1.25(\mathrm{t}, J 7.5 \mathrm{~Hz}, 3 \mathrm{H}) ;{ }^{13} \mathrm{C} \mathrm{NMR}\left(100 \mathrm{MHz}, \mathrm{CD}_{3} \mathrm{CN}\right): \delta$ 169.5, 149.8, 148.0, 138.0, 137.9, 136.1, 133.8, 133.1, 132.2, 130.1, 126.9, $121.5\left(q,{ }^{1} J_{C F}=318.0 \mathrm{~Hz}\right), 115.0,106.2,25.9,25.6,14.7,14.6 ;{ }^{19} \mathrm{~F} \mathrm{NMR}$ 
(377 MHz, CD ${ }_{3} \mathrm{CN}$ ): $\delta$-79.3; HRMS (APCI-positive ionization): calcd for $\mathrm{C}_{17} \mathrm{H}_{18} \mathrm{IO}_{2}\left([\mathrm{M}-\mathrm{OTf}]^{+}\right)$: 381.0351 , found: 381.0348.

2-Carboxyphenyl(4-methoxyphenyl)iodonium triflate (3i). ${ }^{44}$ Reaction of methoxybenzene $\mathbf{2 i}$ (87 $\mathrm{mg}, 0.80$ $\mathrm{mmol}$ ) according to general procedure afforded $101 \mathrm{mg}(100 \%)$ of product $3 \mathbf{i}$ isolated as a gray solid: $\mathrm{mp}$ 213.6-214.9 ${ }^{\circ} \mathrm{C}$ (lit. ${ }^{44}$; mp 195-206 ${ }^{\circ} \mathrm{C}$ ); IR (KBr) cm${ }^{-1}$ 3486, 3093, 2984, 2950, 2851, 1668, 1582, 1492, 1464, $1308,1258,1164,1026,756 ;{ }^{1} \mathrm{H}$ NMR $\left(400 \mathrm{MHz}, \mathrm{CD}_{3} \mathrm{CN}\right): \delta 8.36(\mathrm{~d}, J 8.0 \mathrm{~Hz}, 1 \mathrm{H}), 8.01(\mathrm{~d}, J 8.0 \mathrm{~Hz}, 1 \mathrm{H}), 7.76(\mathrm{t}, J$ $8.0 \mathrm{~Hz}, 1 \mathrm{H}), 7.70(\mathrm{t}, J 8.0 \mathrm{~Hz}, 1 \mathrm{H}), 7.22(\mathrm{~d}, J 8.0 \mathrm{~Hz}, 1 \mathrm{H}), 7.06(\mathrm{~d}, J 8.0 \mathrm{~Hz}, 1 \mathrm{H}), 3.94(\mathrm{~s}, 3 \mathrm{H}) ;{ }^{13} \mathrm{C} \mathrm{NMR}(100 \mathrm{MHz}$, $\mathrm{CD}_{3} \mathrm{CN}$ ): $\delta 169.7,164.8,140.8,137.9,133.8,132.2,130.0,127.0,121.6$ (q, $\left.{ }^{1} J_{C F}=319.5 \mathrm{~Hz}\right), 115.5,97.8,56.4$; HRMS (ESI-positive ionization): calcd for $\mathrm{C}_{14} \mathrm{H}_{12} \mathrm{IO}_{3}\left([\mathrm{M}-\mathrm{OTf}]^{+}\right)$: 354.9831 , found: 354.9833 .

2-Carboxyphenyl(4-ethoxyphenyl)iodonium triflate (3j). Reaction of ethoxybenzene $\mathbf{2 j}$ (98 $\mathrm{mg}, 0.80 \mathrm{mmol}$ ) according to general procedure afforded $100 \mathrm{mg}(97 \%)$ of product $\mathbf{3 j}$ isolated as a gray solid: $\mathrm{mp}$ 214.8-216.0 ${ }^{\circ} \mathrm{C}$; IR $(\mathrm{KBr}) \mathrm{cm}^{-1} 3463,3089,2984,2897,1672,1586,1493,1471,1261,1179,1027,748 ;{ }^{1} \mathrm{H} \mathrm{NMR}(400 \mathrm{MHz}$, $\left.\mathrm{CD}_{3} \mathrm{CN}\right): \delta 8.36(\mathrm{~d}, J 8.0 \mathrm{~Hz}, 1 \mathrm{H}), 7.99(\mathrm{~d}, J 8.0 \mathrm{~Hz}, 2 \mathrm{H}), 7.76(\mathrm{t}, J 8.0 \mathrm{~Hz}, 1 \mathrm{H}), 7.70(\mathrm{t}, J 8.0 \mathrm{~Hz}, 1 \mathrm{H}), 7.19(\mathrm{~d}, J 8.0$ $\mathrm{Hz}, 2 \mathrm{H}), 7.07(\mathrm{~d}, J 8.0 \mathrm{~Hz}, 1 \mathrm{H}), 4.20(\mathrm{q}, J 6.5 \mathrm{~Hz}, 2 \mathrm{H}), 1.44(\mathrm{t}, J 6.5 \mathrm{~Hz}, 3 \mathrm{H}) ;{ }^{13} \mathrm{C} \mathrm{NMR}\left(100 \mathrm{MHz}, \mathrm{DMSO}-d_{6}\right)$ : $\delta 169.3,162.7,140.1,136.7,133.0,131.5,129.7,129.3,121.2$ (q, ${ }^{1} J_{C F}=320.0 \mathrm{~Hz}$ ), 118.8, 116.9, 101.5, 64.4, 14.5; ${ }^{19} \mathrm{~F} \mathrm{NMR} \mathrm{(377} \mathrm{MHz,} \mathrm{CD} \mathrm{CD}_{3} \mathrm{CN}$ ): $\delta$-79.4; HRMS (ESI-positive ionization): calcd for $\mathrm{C}_{15} \mathrm{H}_{14} \mathrm{IO}_{3}\left([\mathrm{M}-\mathrm{OTf}]^{+}\right)$: 368.9988, found: 368.9990 .

2-Carboxyphenyl(2,4,6-trimethoxyphenyl)iodonium triflate (3k). Reaction of 1,3,5-trimethoxybenzene $\mathbf{2 k}$ (135 $\mathrm{mg}, 0.80 \mathrm{mmol}$ ) according to general procedure afforded $72 \mathrm{mg}(64 \%)$ of product 3k isolated as a purple solid: $\mathrm{mp} 167.7^{\circ} \mathrm{C}$ (decomp.); IR (KBr) cm $\mathrm{cm}^{-1} 3445,3100,2954,2913,1672,1585,1470,1418,1291,1164,1028$, 750; ${ }^{1} \mathrm{H}$ NMR (400 MHz, CD ${ }_{3} \mathrm{CN}$ ): $\delta 8.28$ (dd, J $\left.7.6 \mathrm{~Hz}, 1.4 \mathrm{~Hz}, 1 \mathrm{H}\right), 7.69$ (dd, J 7.6 Hz, $\left.1.4 \mathrm{~Hz}, 1 \mathrm{H}\right), 7.66-7.59$ (m, $1 \mathrm{H}), 7.04$ (dd, J $8.4 \mathrm{~Hz}, 1.2 \mathrm{~Hz}, 1 \mathrm{H}), 6.43(\mathrm{~s}, 2 \mathrm{H}), 3.91(\mathrm{~s}, 3 \mathrm{H}), 3.81(\mathrm{~s}, 6 \mathrm{H}) ;{ }^{13} \mathrm{C} \mathrm{NMR}\left(100 \mathrm{MHz}, \mathrm{CD}_{3} \mathrm{CN}\right): \delta 169.8$, $169.3,162.4,137.9,133.9,132.0,128.7,127.3,118.4$ (q, ${ }^{1} J_{C F}=317.3 \mathrm{~Hz}$ ), 113.7, 92.9, 79.7, 57.7, 56.9; ${ }^{19} \mathrm{~F} \mathrm{NMR}$ (377 MHz, CD ${ }_{3} \mathrm{CN}$ ): $\delta$-79.4; HRMS (ESI-positive ionization): calcd for $\mathrm{C}_{16} \mathrm{H}_{16} \mathrm{IO}_{5}$ ([M-OTf] ${ }^{+}$): 415.0042 , found: 415.0026.

Single crystals of product 3k suitable for X-ray crystallographic analysis were obtained by slow evaporation of methylene chloride-ether solution. For details on crystal structure of compound $\mathbf{3 k}$ see the CIF file in Supporting Information. Selected crystallographic data for 3k: Triclinic, $P-1, a=8.1177(2) \AA, b=8.7075(2) \AA, c$ $=14.9429(11) \AA, \alpha=73.353(5), \beta=89.458(6), \gamma=83.352(6), V=1004.85(9) \AA^{3}, Z=2, R(I>2.0 / \sigma(I))=0.0438, R w$ (all) $=0.0582$, CCDC 2021393 .

2-Carboxyphenyl(4-chlorophenyl)iodonium triflate (3l). ${ }^{44}$ Reaction of chlorobenzene $2 \mathrm{l}(90 \mathrm{mg}, 0.80 \mathrm{mmol})$ according to general procedure afforded $78 \mathrm{mg}(77 \%)$ of product $3 \mathbf{3}$ isolated as a white solid: $\mathrm{mp}$ 136.7-138.4 ${ }^{\circ} \mathrm{C}$ (lit. ${ }^{44}$; $\mathrm{mp} \mathrm{218-220}{ }^{\circ} \mathrm{C}$ ); IR (KBr) cm${ }^{-1} 3511,3084,2880,1661,1613,1472,1442,1257,1171,1093,1030,743$; ${ }^{1} \mathrm{H}$ NMR $\left.(400 \mathrm{MHz} \text {, DMSO-d })_{6}\right): \delta 8.03(\mathrm{~d}, J 8.0 \mathrm{~Hz}, 2 \mathrm{H}), 8.00-7.93(\mathrm{~m}, 2 \mathrm{H}), 7.88(\mathrm{~d}, J 8.0 \mathrm{~Hz}, 2 \mathrm{H}), 7.74-7.68(\mathrm{~m}$, $2 \mathrm{H}) ;{ }^{19} \mathrm{~F} \mathrm{NMR} \mathrm{(377} \mathrm{MHz,} \mathrm{CD} 3 \mathrm{CN}$ ): $\delta$-77.7; HRMS (ESI-positive ionization): calcd for $\mathrm{C}_{13} \mathrm{H}_{9}{ }^{35} \mathrm{ClIO}_{2}\left([\mathrm{M}-\mathrm{OTf}]^{+}\right)$: 358.9336, found: 358.9312 .

One-pot preparation of pseudocyclic diaryliodonium triflates $\mathbf{3}$ using 2-iodosylbenzoic acid 4 and benzene $\mathbf{2 a}$ using TfOH. Benzene 2a (62 mg, $0.80 \mathrm{mmol}$ ) and trifluoromethanesulfonic acid ( $66 \mathrm{mg}, 0.44 \mathrm{mmol}$ ) was added to a solution of 2-iodosylbenzoic acids $4(0.20 \mathrm{mmol})$ in dichloromethane $(1 \mathrm{~mL})$. The reaction was stirred at room temperature for 24 hours. After completion of the reaction, the solvent was removed under reduced pressure and the solid product was washed with diethyl ether several times then dried in vacuum to give the pure compound $\mathbf{3 a}, \mathbf{n}, \mathbf{0}$. 
2-Carboxyphenyl(phenyl)iodonium triflate (3a). Reaction of 2-iodosylbenzoic acid $4 a(53 \mathrm{mg}, 0.20 \mathrm{mmol}$ ) according to general procedure afforded $95 \mathrm{mg}$ (100\%) of product $3 a$ isolated as a white solid identical to the same from previous experiment.

4-Methyl-2-carboxyphenyl(phenyl)iodonium triflate (3m). Reaction of 4-methyl-2-iodosylbenzoic acid $\mathbf{4 b}$ (56 $\mathrm{mg}, 0.20 \mathrm{mmol}$ ) according to general procedure afforded $96 \mathrm{mg}(92 \%)$ of product $3 \mathrm{~m}$ isolated as a white solid: $\mathrm{mp} 149.3-152.6^{\circ} \mathrm{C}$; IR (KBr) cm $\mathrm{cm}^{-1} 3410,3087,3061,2928,1660,1574,1448,1257,1167,1033,744 ;{ }^{1} \mathrm{H} N M R$ $\left(400 \mathrm{MHz}, \mathrm{CD}_{3} \mathrm{OD}\right): \delta 8.27-8.20(\mathrm{~m}, 3 \mathrm{H}), 7.92(\mathrm{t}, J 8.0 \mathrm{~Hz}, 1 \mathrm{H}), 7.73(\mathrm{t}, J 8.0 \mathrm{~Hz}, 1 \mathrm{H}), 7.73(\mathrm{t}, J 8.0 \mathrm{~Hz}, 2 \mathrm{H}), 7.75-$ $7.49(\mathrm{~m}, 1 \mathrm{H}), 6.88(\mathrm{~d}, J 8.0 \mathrm{~Hz}, 1 \mathrm{H}), 2.46(\mathrm{~s}, 3 \mathrm{H}) ;{ }^{13} \mathrm{C} N M R\left(125 \mathrm{MHz}, \mathrm{CD}_{3} \mathrm{OD}\right): \delta 169.8,142.5,137.7,137.2$, 133.4, 133.4, 132.2, 129.1, 127.1, $120.4\left(\mathrm{q},{ }^{1} \mathrm{~J}_{\mathrm{CF}}=317 \mathrm{~Hz}\right), 19.21 ;{ }^{19} \mathrm{~F} \mathrm{NMR}\left(377 \mathrm{MHz}, \mathrm{CD}_{3} \mathrm{OD}\right): \delta-80.1 ; \mathrm{HRMS}$ (ESI-positive ionization): calcd for $\mathrm{C}_{14} \mathrm{H}_{12} \mathrm{IO}_{2}\left([\mathrm{M}-\mathrm{OTf}]^{+}\right)$: 338.9882 , found: 338.9900 .

4-Bromo-2-carboxyphenyl(phenyl)iodonium triflate (3n). Reaction of 4-bromo-2-iodosylbenzoic acid $4 \mathbf{c}$ (69 $\mathrm{mg}, 0.2 \mathrm{mmol}$ ) according to general procedure afforded $93 \mathrm{mg}(84 \%)$ of product $3 \mathrm{n}$ isolated as a white solid: $\mathrm{mp} 186.1-187.5^{\circ} \mathrm{C}$; IR (KBr) cm ${ }^{-1} 3463,3080,1663,1620,1553,1445,1256,1172,1024,738 ;{ }^{1} \mathrm{H}$ NMR (400 $\left.\mathrm{MHz}, \mathrm{CD}_{3} \mathrm{CN}\right): \delta 8.48(\mathrm{~d}, J 2.2 \mathrm{~Hz}, 1 \mathrm{H}), 8.12(\mathrm{~d}, J 8.4 \mathrm{~Hz}, 2 \mathrm{H}), 7.99-7.89(\mathrm{~m}, 1 \mathrm{H}), 7.80(\mathrm{dd}, J 9.0 \mathrm{~Hz}, 2.2 \mathrm{~Hz}, 1 \mathrm{H})$, 7.77-7.65 (m, 2H), 6.90 (d, J $9.0 \mathrm{~Hz}, 1 \mathrm{H}) ;{ }^{13} \mathrm{C}$ NMR $\left(100 \mathrm{MHz}, \mathrm{CD}_{3} \mathrm{CN}\right): \delta 168.5,140.4,138.7,136.3,134.8$, 133.4, 131.9, 128.8, 126.3, $121.6\left(q,{ }^{1} J_{C F}=317 \mathrm{~Hz}\right), 113.5,109.8 ;{ }^{19} \mathrm{~F} \mathrm{NMR}\left(377 \mathrm{MHz}, \mathrm{CD}_{3} \mathrm{CN}\right): \delta-79.4 ; \mathrm{HRMS}$ (ESI-positive ionization): calcd for $\mathrm{C}_{13} \mathrm{H}_{9}{ }^{79} \mathrm{BrIO}_{2}$ ([M-OTf $\left.]^{+}\right)$: 402.8831 , found: 338.9885 .

One-pot preparation of pseudocyclic diaryliodonium triflates 3 a using 2 -iodobenzoic acid 5 and benzene $2 \mathrm{a}$ using TfOH with mCPBA. Benzene $2 \mathrm{a}(62 \mathrm{mg}, 0.80 \mathrm{mmol})$, trifluoromethanesulfonic acid (66 mg, $0.44 \mathrm{mmol}$ ), and $m$ CPBA (76 mg, $0.44 \mathrm{mmol}$ ) was added to a solution of 2-iodobenzoic acid 5 (50 mg, $0.20 \mathrm{mmol}$ ) in dichloromethane $(1 \mathrm{~mL})$. The reaction was stirred at room temperature for 24 hours. After completion of the reaction, the solvent was removed under reduced pressure and the solid product was washed with diethyl ether several times then dried in vacuum to give the pure compound 3a; $64 \mathrm{mg}(68 \%)$ isolated as a brown solid identical to the same from previous experiment.

General procedure for preparation of arylbenziodoxolones 6 from pseudocyclic diaryliodonium triflates 3. Pseudocyclic diaryliodonium triflates 3 was added to dichloromethane $(2 \mathrm{~mL})$ and saturated $\mathrm{NaHCO}_{3}(1 \mathrm{~mL})$. The reaction was stirred at room temperature for 1 hour. After completion of the reaction, the solvent was removed under reduced pressure and the solid product was washed with diethyl ether several times then dried in vacuum to give the pure compound 6.

1-Phenyl-1 $\lambda^{3}$-benzo[d][1,2]iodaoxol-3(1H)-one (6a). ${ }^{42}$ Reaction of $3 \mathbf{a}(95 \mathrm{mg}, 0.20 \mathrm{mmol}$ ) according to general procedure afforded $64 \mathrm{mg}(100 \%)$ of product 6 a isolated as a white solid: $\mathrm{mp} 210.1-211.0{ }^{\circ} \mathrm{C}$ (lit. ${ }^{42}$; mp 221 $222^{\circ} \mathrm{C}$ ); IR ( $\left.\mathrm{KBr}\right) \mathrm{cm}^{-1} 3042,1609,1557,1475,1340,738 ;{ }^{1} \mathrm{H} \mathrm{NMR}\left(300 \mathrm{MHz}, \mathrm{CDCl}_{3}\right): \delta 8.40$ (dd, J 7.5 Hz, $1.2 \mathrm{~Hz}$, $1 \mathrm{H}), 8.02(\mathrm{~d}, J 7.8 \mathrm{~Hz}, 2 \mathrm{H}), 7.77(\mathrm{t}, J 7.5 \mathrm{~Hz}, 1 \mathrm{H}), 7.65-7.50(\mathrm{~m}, 3 \mathrm{H}), 7.43-7.32(\mathrm{~m}, 1 \mathrm{H}), 6.74(\mathrm{~d}, J 8.4 \mathrm{~Hz}, 1 \mathrm{H}) ;{ }^{13} \mathrm{C}$ NMR $\left(75 \mathrm{MHz}_{1} \mathrm{CDCl}_{3}\right): \delta 166.6,137.2,133.5,133.4,132.7,132.6,131.8,130.7,126.1,115.6,115.3$; HRMS (ESIpositive ionization): calcd for $\mathrm{C}_{13} \mathrm{H}_{10} \mathrm{IO}_{2}\left([\mathrm{M}+\mathrm{H}]^{+}\right)$: 324.9725 , found: 324.9736 .

1-Mesityl-1 $\lambda^{3}$-benzo[d][1,2]iodaoxol-3(1H)-one (6b). ${ }^{42}$ Reaction of $3 b(103 \mathrm{mg}, 0.20 \mathrm{mmol})$ according to general procedure afforded $65 \mathrm{mg}(89 \%)$ of product $6 \mathbf{b}$ isolated as a white solid: $\mathrm{mp} 222.4-223.1{ }^{\circ} \mathrm{C}$ (lit. ${ }^{42} ; \mathrm{mp}$ $\left.223-223.5^{\circ} \mathrm{C}\right)$; IR (KBr) cm ${ }^{-1} 3058,2974,2948,2920,1616,1558,1441,1034,758 ;{ }^{1} \mathrm{H}$ NMR $\left(300 \mathrm{MHz}, \mathrm{CD}_{3} \mathrm{OD}\right)$ : $\delta 8.31(\mathrm{~d}, J 7.8 \mathrm{~Hz}, 1 \mathrm{H}), 7.72-7.61(\mathrm{~m}, 1 \mathrm{H}), 7.55-7.44(\mathrm{~m}, 1 \mathrm{H}), 7.29(\mathrm{~s}, 2 \mathrm{H}), 6.79(\mathrm{~d}, J 8.1 \mathrm{~Hz}, 1 \mathrm{H}), 2.53(\mathrm{~s}, 6 \mathrm{H})$, $2.43(\mathrm{~s}, 3 \mathrm{H})$; HRMS (ESI-positive ionization): calcd for $\mathrm{C}_{16} \mathrm{H}_{16} \mathrm{IO}_{2}\left([\mathrm{M}+\mathrm{H}]^{+}\right): 367.0195$, found: 367.0210 .

1-(2,5-Dimethylphenyl)-1 $\lambda^{3}$-benzo[d][1,2]iodaoxol-3(1H)-one $(6 \mathrm{c}){ }^{45}$ Reaction of 3c (100 $\left.\mathrm{mg}, 0.20 \mathrm{mmol}\right)$ according to general procedure afforded $70 \mathrm{mg}(100 \%)$ of product $6 \mathrm{c}$ isolated as a light yellow solid: $\mathrm{mp} 110.7-$ $111.8^{\circ} \mathrm{C}$ (lit. ${ }^{45}$; mp $214-214.5^{\circ} \mathrm{C}$ ); IR ( $\left.\mathrm{KBr}\right) \mathrm{cm}^{-1} 3068,2970,2927,1608,1489,1368,1031,750 ;{ }^{1} \mathrm{H}$ NMR (500 
$\left.\mathrm{MHz}, \mathrm{CD}_{3} \mathrm{OD}\right): \delta 8.46(\mathrm{~d}, J 7.5 \mathrm{~Hz}, 1 \mathrm{H}), 7.72-7.65(\mathrm{~m}, 1 \mathrm{H}), 7.56-7.45(\mathrm{~m}, 1 \mathrm{H}), 7.43-7.38(\mathrm{~m}, 2 \mathrm{H}), 7.37-7.29(\mathrm{~m}$, $1 \mathrm{H}), 6.63(\mathrm{~d}, J 8.5 \mathrm{~Hz}, 1 \mathrm{H}), 2.40(\mathrm{~s}, 3 \mathrm{H}), 2.43(\mathrm{~s}, 3 \mathrm{H})$; HRMS (APCl-positive ionization): calcd for $\mathrm{C}_{15} \mathrm{H}_{14} \mathrm{IO}_{2}$ $\left([\mathrm{M}+\mathrm{H}]^{+}\right): 353.0038$, found: 353.0032 .

1-(2,3,5,6-Tetramethylphenyl)-1 $\lambda^{3}$-benzo[d][1,2]iodaoxol-3(1H)-one (6d). ${ }^{42}$ Reaction of $3 d$ (96 mg, 0.18 $\mathrm{mmol}$ ) according to general procedure afforded $56 \mathrm{mg}(82 \%)$ of product $\mathbf{6 d}$ isolated as a white solid: $\mathrm{mp} 218.0$ $220.4{ }^{\circ} \mathrm{C}$ (lit. $^{42}$; mp 221.5-222.5 ${ }^{\circ} \mathrm{C}$ ); IR (KBr) cm ${ }^{-1}$ 3060, 3008, 2963, 2941, 2920, 2850, 1598, 1554, 1466, 1346, 1006, 760; ${ }^{1} \mathrm{H}$ NMR (500 MHz, $\left.\mathrm{CDCl}_{3}\right): \delta 8.51$ (dd, J $\left.8.0 \mathrm{~Hz}, 1.5 \mathrm{~Hz}, 1 \mathrm{H}\right), 7.64-7.58(\mathrm{~m}, 1 \mathrm{H}), 7.43-7.37(\mathrm{~m}, 1 \mathrm{H})$, 7.28-7.24 (m, $1 \mathrm{H}), 7.43-7.38(\mathrm{~m}, 2 \mathrm{H}), 7.37-7.29(\mathrm{~m}, 1 \mathrm{H}), 6.63(\mathrm{~d}, J 8.5 \mathrm{~Hz}, 1 \mathrm{H}), 2.40(\mathrm{~s}, 3 \mathrm{H}), 2.43(\mathrm{~s}, 3 \mathrm{H}) ; \mathrm{HRMS}$ (ESI-positive ionization): calcd for $\mathrm{C}_{17} \mathrm{H}_{18} \mathrm{IO}_{2}\left([\mathrm{M}+\mathrm{H}]^{+}\right)$: 381.0356 , found: 381.0366 .

1-(4-(tert-butyl)phenyl)-1 $\lambda^{3}$-benzo[d][1,2]iodaoxol-3(1H)-one (6e). ${ }^{42}$ Reaction of 3 e $(106 \mathrm{mg}, 0.20 \mathrm{mmol})$ according to general procedure afforded $72 \mathrm{mg}$ (95\%) of product 6e isolated as a white solid: $\mathrm{mp} 230.9-231.4$ ${ }^{\circ} \mathrm{C}$ (lit. ${ }^{42}$; mp 233.5-234 ${ }^{\circ} \mathrm{C}$ ); IR ( $\left.\mathrm{KBr}\right) \mathrm{cm}^{-1}$ 3059, 2959, 2869, 1612, 1556, 1482, 1347, 1058, 748; ${ }^{1} \mathrm{H}$ NMR (500 $\mathrm{MHz}_{\mathrm{CDCl}}$ ): $\delta 8.48(\mathrm{dd}, J 7.8 \mathrm{~Hz}, 1.8 \mathrm{~Hz}, 1 \mathrm{H}), 7.88(\mathrm{~d}, \mathrm{~J} 9.0 \mathrm{~Hz}, 2 \mathrm{H}), 7.64-7.57(\mathrm{~m}, 3 \mathrm{H}), 7.47-7.41(\mathrm{~m}, 1 \mathrm{H}), 6.82$ (d, J $8.0 \mathrm{~Hz}, 1 \mathrm{H}), 1.40(\mathrm{~s}, 9 \mathrm{H})$; HRMS (ESI-positive ionization): calcd for $\mathrm{C}_{17} \mathrm{H}_{18} \mathrm{IO} \mathrm{O}_{2}\left([\mathrm{M}+\mathrm{H}]^{+}\right)$: 381.0356, found: 381.0366.

1-(p-Tolyl)-1 $\lambda^{3}$-benzo[d][1,2]iodaoxol-3(1H)-one (6f). ${ }^{42}$ Reaction of $3 f(88 \mathrm{mg}, 0.18 \mathrm{mmol})$ according to general procedure afforded $42 \mathrm{mg}(69 \%)$ of product 6 f isolated as a white solid: $\mathrm{mp} 195.9-197.7^{\circ} \mathrm{C}\left(\mathrm{lit} .{ }^{42} ; \mathrm{mp}\right.$ $\left.217-218^{\circ} \mathrm{C}\right)$; IR (KBr) cm ${ }^{-1} 3074,2977,2923,1610,1557,1488,1329,747 ;{ }^{1} \mathrm{H} \mathrm{NMR}\left(400 \mathrm{MHz}, \mathrm{CDCl}_{3}\right): \delta 8.49(\mathrm{~d}$, J $8.0 \mathrm{~Hz}, 1 \mathrm{H}), 7.81(\mathrm{~d}, J 8.0 \mathrm{~Hz}, 2 \mathrm{H}), 7.65-7.56(\mathrm{~m}, 1 \mathrm{H}), 7.47-7.37(\mathrm{~m}, 3 \mathrm{H}), 6.78(\mathrm{~d}, J 8.0 \mathrm{~Hz}, 2 \mathrm{H}), 2.52(\mathrm{~s}, 3 \mathrm{H})$; HRMS (APCl-positive ionization): calcd for $\mathrm{C}_{14} \mathrm{H}_{12} \mathrm{IO}_{2}\left([\mathrm{M}+\mathrm{H}]^{+}\right)$: 338.9882, found: 338.9882 .

1-(2,4,6-Triisopropylphenyl)-1 $\lambda^{3}$-benzo[d][1,2]iodaoxol-3(1H)-one $(\mathbf{6 g})$. Reaction of $\mathbf{3 g}(120 \mathrm{mg}, 0.20 \mathrm{mmol})$ according to general procedure afforded $56 \mathrm{mg}$ (62\%) of product $6 \mathrm{~g}$ isolated as a white solid: $\mathrm{mp} 197.9-198.8$ ${ }^{\circ} \mathrm{C}$; IR (KBr) cm ${ }^{-1} 3057,2962,2870,1602,1552,1357,745 ;{ }^{1} \mathrm{H} \mathrm{NMR}\left(300 \mathrm{MHz}, \mathrm{CDCl}_{3}\right): \delta 8.51(\mathrm{~d}, J 7.5 \mathrm{~Hz}, 1 \mathrm{H})$, $7.61(\mathrm{t}, J 7.5 \mathrm{~Hz}, 1 \mathrm{H}), 7.46-7.38(\mathrm{~m}, 1 \mathrm{H}), 7.25(\mathrm{~s}, 2 \mathrm{H}), 6.76(\mathrm{~d}, J 8.1 \mathrm{~Hz}, 1 \mathrm{H}), 3.19$ (sept, J $6.9 \mathrm{~Hz}, 2 \mathrm{H}), 3.02$ (sept, J $6.9 \mathrm{~Hz}, 1 \mathrm{H}), 1.31$ (d, J $6.9 \mathrm{~Hz}, 12 \mathrm{H}), 1.16(\mathrm{~d}, J 6.9 \mathrm{~Hz}, 6 \mathrm{H}) ;{ }^{13} \mathrm{C} \mathrm{NMR}\left(75 \mathrm{MHz}, \mathrm{CDCl}_{3}\right): \delta 166.7,155.0,153.6$, 134.2, 133.5, 133.1, 130.9, 125.4, 123.9, 119.3, 115.2, 37.9, 34.4, 25.1, 23.8; HRMS (APCl-positive ionization): calcd for $\mathrm{C}_{22} \mathrm{H}_{28} \mathrm{IO} \mathrm{O}_{2}\left([\mathrm{M}+\mathrm{H}]^{+}\right): 451.1134$, found: 451.1112 .

1-(3,4-Diethylphenyl)-1 $\lambda^{3}$-benzo[d][1,2]iodaoxol-3(1H)-one (6h). Reaction of $3 \mathrm{~h}$ (99 $\mathrm{mg}, 0.19 \mathrm{mmol}$ ) according to general procedure afforded $47 \mathrm{mg} \mathrm{(66 \% )} \mathrm{of} \mathrm{product} 6 \mathrm{~h}$ isolated as a white solid: $\mathrm{mp}$ 193.8-195.6 ${ }^{\circ} \mathrm{C}$; IR (KBr) cm ${ }^{-1} 3064,2966,2936,2880,1611,1558,1331,740 ;{ }^{1} \mathrm{H} \mathrm{NMR}\left(500 \mathrm{MHz}, \mathrm{CDCl}_{3}\right): \delta 8.42(\mathrm{~d}, J 7.5 \mathrm{~Hz}$, $1 \mathrm{H}), 7.78-7.73(\mathrm{~m}, 2 \mathrm{H}), 7.55(\mathrm{t}, J 7.0 \mathrm{~Hz}, 1 \mathrm{H}), 7.43-7.34(\mathrm{~m}, 2 \mathrm{H}), 6.79(\mathrm{~d}, J 7.0 \mathrm{~Hz}, 1 \mathrm{H}), 2.83-2.71(\mathrm{~m}, 4 \mathrm{H}), 1.31(\mathrm{t}$, J $7.5 \mathrm{~Hz}, 3 \mathrm{H}), 1.28$ (t, J $7.5 \mathrm{~Hz}, 3 \mathrm{H}) ;{ }^{13} \mathrm{C} \mathrm{NMR}\left(100 \mathrm{MHz} \mathrm{CDCl}_{3}\right): \delta 166.3,147.5,146.3,136.5,134.6,133.5,132.9$, 131.7, 130.8, 125.8, 115.5, 111.5, 25.6, 25.5, 14.9, 14.8; HRMS (APCl-positive ionization): calcd for $\mathrm{C}_{17} \mathrm{H}_{18} \mathrm{IO} \mathrm{O}_{2}$ $\left([\mathrm{M}+\mathrm{H}]^{+}\right): 381.0351$, found: 381.0352 .

1-(4-Methoxyphenyl)-1 $\lambda^{3}$-benzo[d][1,2]iodaoxol-3(1H)-one $(\mathbf{6 i}) .^{47}$ Reaction of $3 \mathbf{i}(105 \mathrm{mg}, 0.20 \mathrm{mmol})$ according to general procedure afforded $70 \mathrm{mg}(99 \%)$ of product $6 \mathbf{i}$ isolated as a white solid: $\mathrm{mp} 159.5-160.1$ ${ }^{\circ} \mathrm{C}$ (lit. ${ }^{47}$; mp 212-214 ${ }^{\circ} \mathrm{C}$ ); IR (KBr) cm ${ }^{-1} 3065,2941,2840,1599,1489,1359,1257,748 ;{ }^{1} \mathrm{H} \mathrm{NMR}(400 \mathrm{MHz}$, $\left.\mathrm{CDCl}_{3}\right): \delta 8.49-8.39(\mathrm{~m}, 1 \mathrm{H}), 7.83(\mathrm{~d}, J 8.0 \mathrm{~Hz}, 2 \mathrm{H}), 7.62-7.51(\mathrm{~m}, 1 \mathrm{H}), 7.45-7.34(\mathrm{~m}, 1 \mathrm{H}), 6.81-6.69(\mathrm{~m}, 1 \mathrm{H}), 7.06$ (d, J $8.0 \mathrm{~Hz}, 2 \mathrm{H}), 3.92(\mathrm{~s}, 3 \mathrm{H}) ;{ }^{13} \mathrm{C} \mathrm{NMR}\left(100 \mathrm{MHz}, \mathrm{CDCl}_{3}\right): \delta 163.1,139.0,133.5,132.9,130.8,125.7,117.8$, 115.9, 104.0, 55.7; HRMS (ESI-positive ionization): calcd for $\mathrm{C}_{14} \mathrm{H}_{12} \mathrm{IO} \mathrm{O}_{3}\left([\mathrm{M}+\mathrm{H}]^{+}\right): 354.9831$, found: 354.9847.

1-(4-Ethoxyphenyl)-1 $\lambda^{3}$-benzo[d][1,2]iodaoxol-3(1H)-one (6j). Reaction of $\mathbf{3 j}$ (100 $\left.\mathrm{mg}, 0.19 \mathrm{mmol}\right)$ according to general procedure afforded $60 \mathrm{mg}(86 \%)$ of product $6 \mathbf{j}$ isolated as a light brown solid: $\mathrm{mp} 218.9-219.8{ }^{\circ} \mathrm{C}$; IR $(\mathrm{KBr}) \mathrm{cm}^{-1} 3086,2977,2928,1603,1488,1340,1252,750 ;{ }^{1} \mathrm{H} \mathrm{NMR}\left(400 \mathrm{MHz}, \mathrm{CDCl}_{3}\right): \delta 8.48(\mathrm{~d}, J 8.0 \mathrm{~Hz}, 1 \mathrm{H})$, $7.83(\mathrm{~d}, J 10.0 \mathrm{~Hz}, 1 \mathrm{H}), 7.59(\mathrm{t}, J 8.0 \mathrm{~Hz}, 1 \mathrm{H}), 7.42(\mathrm{t}, J 8.0 \mathrm{~Hz}, 1 \mathrm{H}), 7.05(\mathrm{t}, J 8.0 \mathrm{~Hz}, 1 \mathrm{H}), 6.78(\mathrm{~d}, J 8.0 \mathrm{~Hz}, 2 \mathrm{H})$, 
$4.15(7.42$ (q, J $7.0 \mathrm{~Hz}, 2 \mathrm{H}), 1.50$ (t, J $7.0 \mathrm{~Hz}, 3 \mathrm{H}) ;{ }^{13} \mathrm{C} \mathrm{NMR}\left(100 \mathrm{MHz}, \mathrm{CDCl}_{3}\right): \delta$ 166.5, 162.5, 139.0, 133.5, 132.9, 120.8, 125.6, 118.1, 116.0, 103.7, 64.2, 14.6; HRMS (ESI-positive ionization): calcd for $\mathrm{C}_{15} \mathrm{H}_{14} \mathrm{IO}_{3}$ $\left([\mathrm{M}+\mathrm{H}]^{+}\right): 368.9988$, found: 368.9999 .

1-(2,4,6-Trimethoxyphenyl)-1 $\lambda^{3}$-benzo[d][1,2]iodaoxol-3(1H)-one (6k). Reaction of 3k (66 mg, $\left.0.12 \mathrm{mmol}\right)$ according to general procedure afforded $44 \mathrm{mg}(89 \%)$ of product $6 \mathbf{k}$ isolated as a white solid: $\mathrm{mp} 249.2-250.3$ ${ }^{\circ} \mathrm{C}$; IR (KBr) cm ${ }^{-1} 3100,3025,2900,1615,1460,1333,1238,752 ;{ }^{1} \mathrm{H} \mathrm{NMR}\left(400 \mathrm{MHz}, \mathrm{CDCl}_{3}\right): \delta 8.45(\mathrm{~d}, \mathrm{~J} 8.0 \mathrm{~Hz}$, $1 \mathrm{H}), 7.42-7.32(\mathrm{~m}, 1 \mathrm{H}), 6.81(\mathrm{~d}, J 8.0 \mathrm{~Hz}, 1 \mathrm{H}), 6.26(\mathrm{~s}, 2 \mathrm{H}), 3.94(\mathrm{~s}, 3 \mathrm{H}), 3.83(\mathrm{~s}, 6 \mathrm{H}) ;{ }^{13} \mathrm{C} \mathrm{NMR}\left(100 \mathrm{MHz}, \mathrm{CDCl}_{3}\right)$ : $\delta 166.9,162.1,134.0,133.02,132.7,130.3,124.7,115.0,91.0,88.3,56.6,55.9,30.9$; HRMS (ESI-positive ionization): calcd for $\mathrm{C}_{16} \mathrm{H}_{16} \mid \mathrm{O}_{5}\left([\mathrm{M}+\mathrm{H}]^{+}\right)$: 415.0042 , found: 415.0047 .

1-(4-Chlorophenyl)-1 $\lambda^{3}$-benzo[d][1,2]iodaoxol-3(1H)-one (6l). ${ }^{42}$ Reaction of 31 (52 $\left.\mathrm{mg}, 0.10 \mathrm{mmol}\right)$ according to general procedure afforded $10 \mathrm{mg}(28 \%)$ of product $6 \mathrm{l}$ isolated as a white solid: $\mathrm{mp} 207.7-208.3^{\circ} \mathrm{C}$ (lit. ${ }^{42}$;

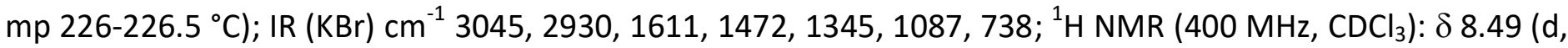
J $8.0 \mathrm{~Hz}, 1 \mathrm{H}), 7.90(\mathrm{~d}, J 8.0 \mathrm{~Hz}, 2 \mathrm{H}), 7.69-7.61(\mathrm{~m}, 1 \mathrm{H}), 7.58(\mathrm{~d}, J 8.0 \mathrm{~Hz}, 2 \mathrm{H}), 7.51-7.42(\mathrm{~m}, 1 \mathrm{H}), 6.77(\mathrm{~d}, J 8.0 \mathrm{~Hz}$, $1 \mathrm{H})$; HRMS (ESI-positive ionization): calcd for $\mathrm{C}_{13} \mathrm{H}_{9}{ }^{35} \mathrm{ClIO}_{2}\left([\mathrm{M}+\mathrm{H}]^{+}\right): 358.9336$, found: 358.9835 .

5-Methyl-1-phenyl-1 $\lambda^{3}$-benzo[d][1,2]iodaoxol-3(1H)-one $(6 \mathrm{~m}) .^{50}$ Reaction of $3 \mathrm{~m}(88 \mathrm{mg}, 0.18 \mathrm{mmol})$ according to general procedure afforded $47 \mathrm{mg}$ (78\%) of product $6 \mathrm{~m}$ isolated as a white solid: $\mathrm{mp} 235.6-236.8$ ${ }^{\circ} \mathrm{C}$ (lit. ${ }^{50}$; mp 222-223 ${ }^{\circ} \mathrm{C}$ ); IR (KBr) cm $\mathrm{cm}^{-1} 3049,2966,1618,1454,1338,742 ;{ }^{1} \mathrm{H} \mathrm{NMR}\left(400 \mathrm{MHz}, \mathrm{CDCl}_{3}\right): \delta 8.09(\mathrm{~s}$, $1 \mathrm{H}), 8.03(\mathrm{~d}, J 8.0 \mathrm{~Hz}, 2 \mathrm{H}), 7.78(\mathrm{t}, J 8.0 \mathrm{~Hz}, 2 \mathrm{H}), 7.60(\mathrm{t}, J 8.0 \mathrm{~Hz}, 2 \mathrm{H}), 7.29(\mathrm{~d}, J 8.0 \mathrm{~Hz}, 1 \mathrm{H}), 6.67(\mathrm{~d}, J 8.0 \mathrm{~Hz}$, $1 \mathrm{H}), 2.41(\mathrm{~s}, 3 \mathrm{H})$; HRMS (ESI-positive ionization): calcd for $\mathrm{C}_{14} \mathrm{H}_{12} \mathrm{IO}_{2}\left([\mathrm{M}+\mathrm{H}]^{+}\right)$: 338.9882, found: 338.9865.

5-Bromo-1-phenyl-1 $\lambda^{3}$-benzo[d][1,2]iodaoxol-3(1H)-one (6n). ${ }^{47}$ Reaction of $3 \mathrm{n}$ (30 $\mathrm{mg}, 0.054 \mathrm{mmol}$ ) according to general procedure afforded $12 \mathrm{mg}(55 \%)$ of product $6 \mathrm{n}$ isolated as a white solid: $\mathrm{mp} 243.8-244.4{ }^{\circ} \mathrm{C}$ (lit. ${ }^{47}$; $\left.\mathrm{mp} 234-236{ }^{\circ} \mathrm{C}\right)$; IR (KBr) cm ${ }^{-1} 3045,2955,1621,1443,1382,739 ;{ }^{1} \mathrm{H} \mathrm{NMR}\left(400 \mathrm{MHz}, \mathrm{CDCl}_{3}\right): \delta 8.61(\mathrm{~d}, J 3.0 \mathrm{~Hz}$, $1 \mathrm{H}), 7.97(\mathrm{~d}, J 8.4 \mathrm{~Hz}, 2 \mathrm{H}), 7.80(\mathrm{t}, J 7.6 \mathrm{~Hz}, 1 \mathrm{H}), 7.68-7.57(\mathrm{~m}, 2 \mathrm{H}), 7.52(\mathrm{dd}, J 8.6 \mathrm{~Hz}, 3.0 \mathrm{~Hz}, 1 \mathrm{H}), 6.58(\mathrm{~d}, J 8.6$ $\mathrm{Hz}, 1 \mathrm{H})$; HRMS (ESI-positive ionization): calcd for $\mathrm{C}_{13} \mathrm{H}_{9}{ }^{79} \mathrm{BrIO}_{2}\left([\mathrm{M}+\mathrm{H}]^{+}\right): 402.8831$, found: 402.8805 .

\section{Acknowledgements}

This work was supported by a research grant from the Russian Science Foundation (RSF-16-13-10081-P) and National Science Foundation (CHE-1759798). A.S. is thankful to JSPS Fund for the Promotion of Joint International Research (Grant No 16KK0199) and JST CREST (No. JRMJCR19R2). Some research was carried out using the core facilities of TPU's "Physical and chemical methods of analysis

\section{Supplementary Material}

NMR spectra $(1 \mathrm{H}, 13 \mathrm{C}$ and $19 \mathrm{~F})$ of products can be found in the supplementary material file.

\section{References}

1. Olofsson, B.; Ilan, M.; Rappoport, Z. Patai's The Chemistry of Hypervalent Halogen Compounds; John Wiley \& Sons: Chichester, 2019.

2. Wirth, T.; Ed. Top. Curr. Chem. 2016, 373, 1. 
3. Zhdankin, V. V. Hypervalent lodine Chemistry: Preparation, Structure and Synthetic Application of Polyvalent lodine Compounds; John Wiley \& Sons Ltd: 2014.

https://doi.org/10.1002/9781118341155

4. Parra, A. Chem. Rev. 2019, 119, 12033.

https://doi.org/10.1021/acs.chemrev.9b00338

5. Muniz, K. Acc. Chem. Res. 2018, 51, 1507. https://doi.org/10.1021/acs.accounts.8b00137

6. Yoshimura, A.; Zhdankin, V. V. Chem. Rev. 2016, 116, 3328.

https://doi.org/10.1021/acs.chemrev.5b00547

7. Le Vaillant, F.; Waser, J. Chem. Sci. 2019, 10, 8909.

https://doi.org/10.1039/C9SC03033F

8. Hari, D. P.; Caramenti, P.; Waser, J. Acc. Chem. Res. 2018, 51, 3212.

https://doi.org/10.1021/acs.accounts.8b00468

9. Wang, X.; Studer, A. Acc. Chem. Res. 2017, 50, 1712.

https://doi.org/10.1021/acs.accounts.7b00148

10. Hyatt, I. F. D.; Dave, L.; David, N.; Kaur, K.; Medard, M.; Mowdawalla, C. Org. Biomol. Chem. 2019, 17, 7822.

https://doi.org/10.1039/C90B01267B

11. Charpentier, J.; Fruh, N.; Togni, A. Chem. Rev. 2015, 115, 650.

https://doi.org/10.1021/cr500223h

12. Merritt, E. A.; Olofsson, B. Angew. Chem., Int. Ed. 2009, 48, 9052.

https://doi.org/10.1002/anie.200904689

13. Aradi, K.; Toth, B. L.; Tolnai, G. L.; Novak, Z. Synlett 2016, 27, 1456.

https://doi.org/10.1055/s-0035-1561369

14. Stuart, D. R. Chem. - Eur. J. 2017, 23, 15852.

https://doi.org/10.1002/chem.201702732

15. Fananas-Mastral, M. Synthesis 2017, 49, 1905.

https://doi.org/10.1055/s-0036-1589483

16. Wang, M.; Chen, S.; Jiang, X. Chem. - Asian J. 2018, 13, 2195.

https://doi.org/10.1002/asia.201800609

17. Yusubov, M. S.; Svitich, D. Y.; Larkina, M. S.; Zhdankin, V. V. Arkivoc 2013, (i), 364.

https://doi.org/10.3998/ark.5550190.p008.225

18. Yoshimura, A.; Saito, A.; Zhdankin, V. V. Chem. - Eur. J. 2018, 24, 15156.

https://doi.org/10.1002/chem.201802111

19. Stuart, D. R. Synlett 2017, 28, 275.

https://doi.org/10.1055/s-0036-1588683

20. Yoshimura, A.; Yusubov, M. S.; Zhdankin, V. V. Org. Biomol. Chem. 2016, 14, 4771. https://doi.org/10.1039/C6OB00773B

21. Zhdankin, V. V.; Protasiewicz, J. D. Coord. Chem. Rev. 2014, 275, 54. https://doi.org/10.1016/i.ccr.2014.04.007

22. Brantley, J. N.; Samant, A. V.; Toste, F. D. ACS Cent. Sci. 2016, 2, 341.

https://doi.org/10.1021/acscentsci.6b00119

23. Wang, Z.; Jiang, L.; Sarro, P.; Suero, M. G. J. Am. Chem. Soc. 2019, 141, 15509.

https://doi.org/10.1021/acscentsci.6b00119 
24. Qurban, J.; Elsherbini, M.; Alharbi, H.; Wirth, T. Chem. Commun. 2019, 55, 7998. https://doi.org/10.1039/C9CC03905H

25. Yudasaka, M.; Maruyama, T.; Yamaguchi, E.; Tada, N.; Itoh, A. Eur. J. Org. Chem. 2018, 2018, 550. https://doi.org/10.1002/ejoc.201701521

26. Watanabe, K.; Moriyama, K. J. Org. Chem. 2018, 83, 14827. https://doi.org/10.1021/acs.joc.8b02676

27. Wang, Z.; Herraiz, A. G.; del Hoyo, A. M.; Suero, M. G. Nature 2018, 554, 86. https://doi.org/10.1038/nature25185

28. Vlasenko, Y. A.; Postnikov, P. S.; Trusova, M. E.; Shafir, A.; Zhdankin, V. V.; Yoshimura, A.; Yusubov, M. S. J. Org. Chem. 2018, 83, 12056. https://doi.org/10.1021/acs.joc.8b01995

29. Boelke, A.; Lork, E.; Nachtsheim, B. J. Chem. - Eur. J. 2018, 24, 18653. https://doi.org/10.1002/chem.201804957

30. Yoshimura, A.; Fuchs, J. M.; Middleton, K. R.; Maskaev, A. V.; Rohde, G. T.; Saito, A.; Postnikov, P. S.; Yusubov, M. S.; Nemykin, V. N.; Zhdankin, V. V. Chem. - Eur. J. 2017, 23, 16738. https://doi.org/10.1002/chem.201704393

31. Yoshimura, A.; Klasen, S. C.; Shea, M. T.; Nguyen, K. C.; Rohde, G. T.; Saito, A.; Postnikov, P. S.; Yusubov, M. S.; Nemykin, V. N.; Zhdankin, V. V. Chem. - Eur. J. 2017, 23, 691. https://doi.org/10.1002/chem.201604475

32. Geary, G. C.; Hope, E. G.; Singh, K.; Stuart, A. M. RSC Adv. 2015, 5, 16501. https://doi.org/10.1039/C4RA15733H

33. Hamnett, D. J.; Moran, W. J. Org. Biomol. Chem. 2014, 12, 4156. https://doi.org/10.1039/C4OB00556B

34. Zhu, C.; Yoshimura, A.; Solntsev, P.; Ji, L.; Wei, Y.; Nemykin, V. N.; Zhdankin, V. V. Chem. Commun. 2012, $48,10108$.

https://doi.org/10.1039/c2cc35708a

35. Zhu, C.; Yoshimura, A.; Ji, L.; Wei, Y.; Nemykin, V. N.; Zhdankin, V. V. Org. Lett. 2012, 14, 3170. https://doi.org/10.1021/ol301268j

36. Yoshimura, A.; Nemykin, V. N.; Zhdankin, V. V. Chem.--Eur. J. 2011, 17, 10538. https://doi.org/10.1002/chem.201102265

37. Yoshimura, A.; Nguyen, K. C.; Klasen, S. C.; Saito, A.; Nemykin, V. N.; Zhdankin, V. V. Chem. Commun. 2015, $51,7835$.

https://doi.org/10.1039/C5CC02009C

38. Yoshimura, A.; Nguyen, K. C.; Rohde, G. T.; Saito, A.; Yusubov, M. S.; Zhdankin, V. V. Adv. Synth. Catal. 2016, 358, 2340.

https://doi.org/10.1002/adsc.201600331

39. Yoshimura, A.; Zhdankin, V. V. Arkivoc (iii), 2017, 32. https://doi.org/10.24820/ark.5550190.p009.893

40. Yoshimura, A.; Nguyen, K. C.; Klasen, S. C.; Postnikov, P. S.; Yusubov, M. S.; Saito, A.; Nemykin, V. N.; Zhdankin, V. V. Asian J. Org. Chem. 2016, 5, 1128.

https://doi.org/10.1002/ajoc.201600247

41. Merritt, E. A.; Olofsson, B. Eur. J. Org. Chem. 2011, 3690.

https://doi.org/10.1002/ejoc.201100360

42. Yusubov, M. S.; Yusubova, R. Y.; Nemykin, V. N.; Zhdankin, V. V. J. Org. Chem. 2013, 78, 3767. 
https://doi.org/10.1021/jo400212u

43. Guilbault, A.-A.; Legault, C. Y. ACS Catal. 2012, 2, 219.

https://doi.org/10.1021/cs200612s

44. Kitamura, T.; Nagata, K.; Taniguchi, H. Tetrahedron Lett. 1995, 36, 1081.

https://doi.org/10.1016/0040-4039(94)02459-O

45. Yusubov, M. S.; Soldatova, N. S.; Postnikov, P. S.; Valiev, R. R.; Svitich, D. Y.; Yusubova, R. Y.; Yoshimura, A.;

Wirth, T.; Zhdankin, V. V. Eur. J. Org. Chem. 2018, 2018, 640.

https://doi.org/10.1002/ejoc.201701595

46. Zhdankin, V. V.; Koposov, A. Y.; Su, L. S.; Boyarskikh, V. V.; Netzel, B. C.; Young, V. G. Org. Lett. 2003, 5, 1583.

https://doi.org/10.1021/ol0344523

47. Almasalma, A. A.; Mejia, E. Eur. J. Org. Chem. 2018, 2018, 188.

48. Bertho, S.; Rey-Rodriguez, R.; Colas, C.; Retailleau, P.; Gillaizeau, I. Chem. - Eur. J. 2017, 23, 17674. https://doi.org/10.1002/chem.201704499

49. Lu, B.; Wu, J.; Yoshikai, N. J. Am. Chem. Soc. 2014, 136, 11598. https://doi.org/10.1021/ja5059795

50. Del Mazza, D.; Reinecke, M. G. J. Org. Chem. 1988, 53, 5799. https://doi.org/10.1021/jo00260a001 